\title{
Modificações químicas em solo solarizado, com e sem incorporação de resíduos orgânicos
}

\section{Chemical modifications in solarized soil with addition of organic residues}

\author{
Juliana Cristina Sodário Cruz ${ }^{1 *}$; Glauber José de Castro Gava²; \\ Marli Teixeira de Almeida Minhoni ${ }^{3}$; Nilton Luiz de Souza ${ }^{3}$; Marcelo de Almeida \\ Silva $^{2}$; Elisangela Marques Jeronimo ${ }^{2}$; Carlos Roberto Padovani ${ }^{4}$
}

\section{Resumo}

A solarização de solo é empregada em pequenas propriedades no controle alternativo de fitopatógenos e plantas invasoras, podendo também afetar a disponibilidade de alguns nutrientes e elementos fitotóxicos no solo. Desta forma elaborou-se o ensaio com delineamento fatorial $2 \times 2 \times 4$ (solo solarizado e não solarizado $\mathrm{x}$ com e sem adição de resíduo vegetal x 4 épocas de coleta) para o acompanhamento das variações de $\mathrm{pH}$, matéria orgânica, fósforo, nitrogênio total, potássio, cálcio, magnésio e alumínio em solo caracterizado como Latossolo Vermelho Distrófico, em Botucatu (SP). O resíduo vegetal incorporado foi constituído de restos culturais de couve (Brassica oleraceae var. acephala L.) frescos e triturados. Amostras de solo de cada tratamento foram coletadas de zero a $0,1 \mathrm{~m}$ de profundidade, aos sete, 21,35 e 49 dias de solarização, entre os meses de janeiro a março de 2001. Foram realizadas análises químicas das amostras de solo bem como o monitoramento das temperaturas do solo nos tratamentos. Os tratamentos solarizados com adição de resíduos orgânicos de couve apresentaram maiores índices de $\mathrm{pH}$ e maiores teores de matéria orgânica, nitrogênio total, fósforo, potássio, cálcio, magnésio e menores de alumínio.

Palavras-chave: Controle alternativo, matéria orgânica, fertilidade do solo

\begin{abstract}
The soil solarization is used in small properties as an alternative for control of phytopathogens and weeds, besides it also can affect the availability of some nutrients and phytotoxic elements in the soil. Thus, one experiment was carried out as a three-factor factorial (solarized and not solarized soil $\mathrm{x}$ with and without addition of vegetable residue $\mathrm{x}$ four collection times) for evaluation of variations of $\mathrm{pH}$, organic matter, phosphorus, total nitrogen, potassium, calcium, magnesium and aluminum in a soil characterized as Distrofic Red Latosoil, in Botucatu county (SP). The incorporated vegetable residue was constituted of crop remains of kale (Brassica oleraceae var. acephala L.) fresh and triturated. Soil samples of each treatment were collected from zero to $0.1 \mathrm{~m}$ of depth, at 7,21, 35, and 49 days after solarization treatment, from January to March of 2001. Soil chemical analyses were done as well as the soil temperature were monitored on the treatments. The solarized soil with kale organic residues addition treatment presented higher values of $\mathrm{pH}$, organic matter, total nitrogen, phosphorus, potassium, calcium, magnesium and minor values of aluminum.
\end{abstract}

Key words: Alternative control, organic matter, fertility of soil

1 Bacharel em Engenharia Agronômica-UNESP-Botucatu (SP), Mestrado em Agronomia, Área de Concentração: Proteção de Plantas-UNESP-Botucatu (SP), Doutorado em Agronomia, Área de Concentração: Proteção de Plantas-UNESP-Botucatu (SP). Pesquisadora APTA Pólo Centro Oeste, Jaú, SP. E-mail: cruzjcs@apta.sp.gov.br

2 Pesquisadores APTA Pólo Centro Oeste, Jaú, SP.

3 Professores da Faculdade de Ciências Agronômicas, UNESP, Departamento de Produção Vegetal, Defesa Fitossanitária, Fazenda Experimental Lageado, Botucatu, SP.

4 Professor do Instituto de Biociências, Departamento de Bioestatística, UNESP, 18618-600, Botucatu, SP.

* Autor para correspondência 


\section{Introdução}

Com a descoberta dos efeitos deletérios à camada de ozônio para a humanidade, causada pela utilização de brometo de metila $\left(\mathrm{CH}_{3} \mathrm{Br}\right)$, é cada vez maior a procura por métodos alternativos de manejo que sejam eficientes e economicamente viáveis para a desinfestação de solos e substratos.

Uma das opções é a solarização de solos, que atua na erradicação ou diminuição da concentração de alguns organismos indesejáveis, como plantas daninhas e fitopatógenos antes ou após o plantio (STAPLETON; DEVAY, 1986), sendo comumente utilizado para culturas anuais de ciclo curto e em alguns casos para culturas perenes (KATAN, 1981).

Katan e Devay (1991) definem solarização como uma técnica na qual o solo é umedecido e coberto com filme plástico transparente e posteriormente exposto à luz do sol, durante os meses do ano com maior intensidade de radiação solar. A técnica geralmente é utilizada em pequenas áreas em campo aberto, mas também pode ser aplicada em casas de vegetação ou túneis plásticos, onde neste caso, o período de tratamento pode ser reduzido (MARQUE; SOUZA; CUTOLO-FILHO, 2002).

Uma das vantagens da solarização é o seu efeito residual, devido à não produção do "vácuo biológico", que permite a sobrevivência de alguns microrganismos de solo, pertencentes à comunidade bacteriana e fúngica do mesmo (CRUZ et al., 2005). Como ocorre com alguns antagonistas que impedem ou retardam a reinfestação do solo por fitopatógenos, como por exemplo, Pseudomonas spp. (BUENO; SOUZA, 2003) e Rhizobium spp. (RICCI et al., 2000).

Além do controle de organismos indesejáveis, a solarização pode também alterar algumas propriedades químicas do solo, como foi observado por Ghini et al. (2003) e Patrício (2004). Estes autores verificaram aumento de $\mathrm{NH}_{4}^{+}, \mathrm{Mn}, \mathrm{NO}_{3}^{-}, \mathrm{Mg}^{2+}, \mathrm{K}^{+}$ e da saturação de bases, além da redução de $(\mathrm{H}+\mathrm{Al})$, $\mathrm{Cu}, \mathrm{Fe}$ e $\mathrm{Zn}$ nos solos solarizados, mostrando que a técnica pode interferir na ciclagem de nutrientes bem como na produção de algumas culturas. Entretanto os processos envolvidos na ciclagem são desconhecidos e pouco conclusivos.

Em alguns casos é possível a combinação da solarização com a incorporação de resíduos culturais como fonte de matéria orgânica, onde é obtida a biofumigação, que pode potencializar os efeitos benéficos da técnica, como aumento da disponibilidade de alguns nutrientes (STAPLETON; DEVAY, 1995).

Quando a técnica é associada à adição de alguma fonte de matéria orgânica, esta é decomposta de forma mais rápida, liberando gases e substâncias nocivas que ficam retidas por algum tempo sob o filme plástico, favorecendo o processo de desinfestação.

A combinação da adição de resíduos vegetais de brássicas com a solarização promove melhora no controle de fitopatógenos e na produção de diversas culturas (GAMLIEL; AUSTERWEIL; KRITZMAN, 2000). Neste contexto Bueno et al. (2004), Ambrósio, Bueno e Souza (2004) e Souza e Bueno (2003) verificaram que a incorporação de resíduos desses vegetais em solos solarizados foi eficiente no controle de fitopatógenos, o que justifica seu uso.

Dessa forma, o objetivo do presente trabalho foi monitorar as variações dos níveis de $\mathrm{pH}$ e teores de matéria orgânica, fósforo, nitrogênio total, potássio, cálcio, magnésio e alumínio, em áreas solarizadas e não solarizadas, com ou sem incorporação de resíduos orgânicos de couve (Brassica oleraceae var. acephala L.).

\section{Material e Métodos}

O experimento foi instalado na Fazenda Experimental Lageado, UNESP-Botucatu (SP) (latitude $22^{\circ} 51^{\prime} \mathrm{S}$ e longitude $48^{\circ} 26^{\prime} \mathrm{W}$ ) em um Latossolo Vermelho Distrófico textura média (CARVALHO; ESPINDOLA; PACCOLA, 1983), segundo os critérios da EMBRAPA (1999) que se encontrava, na ocasião, em pousio. 
As características químicas e físicas do solo, antes da implantação do experimento no campo, foram analisadas no Departamento de Recursos Naturais/ Ciências dos Solos da FCA-UNESP-Botucatu (SP), apresentando na camada de 0 a $0,1 \mathrm{~m}: \mathrm{pH}\left(\mathrm{CaCl}_{2}\right)$ 4,6; matéria orgânica $14 \mathrm{~g} \mathrm{dm}^{-3}$; fósforo (resina) 3,0 $\mathrm{mg} \mathrm{dm}{ }^{-3} ;(\mathrm{H}+\mathrm{Al})$ de $25 \mathrm{mmol}_{\mathrm{c}} \mathrm{dm}^{-3}$; potássio 0,4 $\mathrm{mmol}_{\mathrm{c}} \mathrm{dm}^{-3}$; cálcio $9,0 \mathrm{mmol}_{\mathrm{c}} \mathrm{dm}^{-3}$; magnésio 2,0 $\mathrm{mmol}_{\mathrm{c}} \mathrm{dm}^{-3}$; CTC de $37 \mathrm{mmol}_{\mathrm{c}} \mathrm{dm}^{-3}$ e V (\%) 32, respectivamente, segundo Raij et al. (2001).

A área foi previamente preparada realizando-se capina e, em seguida, a retirada dos restos vegetais e nivelamento da mesma.

Foram preparadas duas áreas de $16 \times 3 \mathrm{~m}$, totalizando $48 \mathrm{~m}^{2}$ cada uma. Cada área foi dividida em seis partes de $6 \mathrm{~m}^{2}$ (tratamentos solarizados, constituindo parcelas destrutivas em decorrência do rompimento do plástico no momento de coleta de solo). Uma das partes foi destinada exclusivamente à acomodação de sensores térmicos de solo e outras duas (não solarizadas de $12 \mathrm{~m}^{2}$ cada) que serviram de tratamentos testemunhas. Cada área foi separada por um corredor de um metro de largura.

O estudo das variáveis avaliadas em função da solarização, adição de resíduo vegetal de couve e época de coletas foi realizado segundo análise de variância para o esquema fatorial $2 \times 2 \times 4$ (solo solarizado e não solarizado x com e sem incorporação de resíduos orgânicos de couve $\mathrm{x}$ quatro épocas de coleta) no modelo experimental inteiramente casualizado (MONTEGOMERY, 1991), com três repetições. Desta forma o trabalho apresentou quatro tratamentos: T1-solo não solarizado (NS), T2-solo solarizado (S), T3-solo não solarizado com acréscimo de resíduos orgânicos de couve (NSMO) e T4-solo solarizado com acréscimo de resíduos orgânicos de couve (SMO).

Os resíduos orgânicos de couve (após colheita de uma área comercial da região de Botucatu -SP, diferente do local de montagem do ensaio) foi escolhido devido a sua capacidade de liberar gases voláteis (glucosinolatos), quando em decomposição, que apresentam atividade biocida (ROSA; HEANEY; FENWICK, 1997).

A liberação desses gases aliada a altas temperaturas promovem maior desinfestação de solo. Este material foi incorporado com a utilização de uma enxada rotativa, momentos antes da colocação do filme plástico, na quantidade de $4 \mathrm{~kg} \mathrm{~m}^{-2}$ (conforme a disponibilidade do material) em uma profundidade de até $0,1 \mathrm{~m}$. Seus teores de nitrogênio, fósforo, potássio, cálcio, magnésio e enxofre foram respectivamente de $28 ; 4,5 ; 38 ; 15 ; 5,9$ e $6,6 \mathrm{~g} \mathrm{~kg}^{-1} \mathrm{e}$ uma relação $\mathrm{C} / \mathrm{N}$ de 15,8 , conforme dados apresentados após análise foliar realizada junto ao Departamento de Recursos Naturais/Ciências do Solo-UNESP-Botucatu, SP, segundo Malavolta, Vitti e Oliveira (1997).

Nos tratamentos que receberam a solarização, o solo foi umedecido, até sua capacidade de campo, e imediatamente coberto com filme plástico transparente de polietileno, aditivado contra raios ultravioleta e $150 \mu \mathrm{m}$ de espessura, durante 50 dias.

De cada área foram coletadas 3 amostras compostas, em 10 pontos ao acaso, para cada tratamento e cada época de coleta de solo, compondo as 3 repetições, de 0 a $0,1 \mathrm{~m}$ de profundidade, com a primeira coleta realizada após sete dias de solarização e as posteriores, em intervalos de quatorze dias, nos meses de janeiro a março de 2001 (7, 21, 35 e 49 dias após instalação do experimento no campo).

As amostras foram coletadas com o auxílio de uma pá e régua, após pequenas incisões no filme plástico, com o uso de um estilete, o qual foi posteriormente vedado com fita adesiva. As amostras compostas coletadas no campo foram acondicionadas em sacos plásticos transparentes e conduzidas ao Departamento de Solos e Nutrição de Plantas da ESALQ-USP-Piracicaba-SP para a realização das análises químicas.

Para a determinação das características químicas dos solos de cada tratamento foram usadas as seguintes técnicas: colorimetria para a determinação de matéria orgânica e para fósforo a extração com 
resina trocadora de íons. Para a determinação dos níveis de potássio foi utilizado fotometria de chama de emissão. Os resultados das análises de cálcio e magnésio foram obtidos por espectrofotometria de absorção atômica e de alumínio através de espectrofotometria, a determinação de nitrogênio total foi realizada através da digestão sulfúrica, em digestor Kjeldahl e titulação em ácido sulfúrico a $0,05 \mathrm{~N}$, as metodologia utilizadas foram baseadas em Raij et al. (2001).

A temperatura do solo, de cada tratamento, foi registrada, diariamente por meio de sensores térmicos conectados a um Datalloger DL 2E (coletor de dados modelo CR 23X (Campbell Scientific)), colocados a $0,1 \mathrm{~m}$ de profundidade para cada tratamento. Os cabos destes sensores foram conduzidos até o coletor de dados, sob a borda enterrada do plástico, para evitar danos ao mesmo. As temperaturas foram registradas a cada hora durante o período de solarização.
Todos os resultados foram submetidos à análise de variância, sendo as médias dos tratamentos comparadas pelo teste de Tukey $(\mathrm{p}=0,05)$, quando na presença de interação significativa entre os fatores avaliados. Os processamentos dos resultados, obtidos nas análises, foram realizados utilizando-se o programa estatístico SAEG 4.0, da Universidade Federal de Viçosa - Departamento de Informática.

\section{Resultados e Discussão}

Durante a realização do experimento o valor médio de temperatura do ar observada foi de $23,6^{\circ} \mathrm{C}$, enquanto que a umidade relativa foi de 81,4 . A precipitação média, durante o período de duração do experimento, foi de $10,8 \mathrm{~mm}$, sendo diariamente registrada, como apresentado na Figura 1, segundo os dados obtidos junto ao Departamento de Recursos Naturais/Ciências Ambientais-UNESP-Botucatu (fonte: CRUZ, 2003).

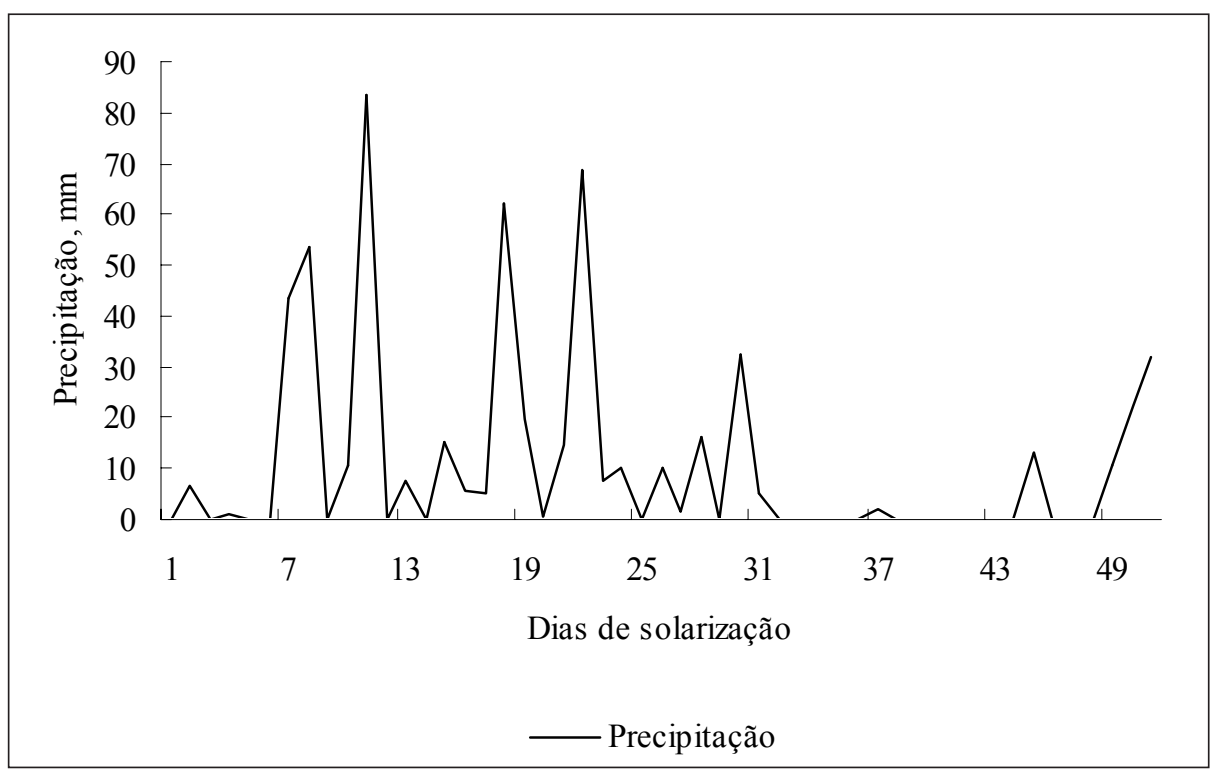

Figura 1. Precipitação, em mm, na região da área experimental, ao longo do período de solarização.

Com relação ao solo, o tratamento não solarizado (NS) apresentou temperaturas médias máximas de $34,3^{\circ} \mathrm{C}$ e médias mínimas de $21,4^{\circ} \mathrm{C}$; enquanto que o solarizado (S) apresentou respectivamente $40,6^{\circ} \mathrm{C}$ e $25,7 \mathrm{C}$; para o tratamento não solarizado com adição de resíduos orgânicos (NSMO), $33,6^{\circ} \mathrm{C}$ e $25,7^{\circ} \mathrm{C}$ e finalmente, para o solarizado com adição de resíduos orgânicos (SMO) $48,8^{\circ} \mathrm{C}$ e $31,9^{\circ} \mathrm{C}$, como mostra a Figura 2 (fonte: CRUZ, 2003).

O tratamento solarizado com adição de resíduos orgânicos (SMO) foi o que apresentou maiores temperaturas, quando comparado ao tratamento sem 
solarização e sem acréscimo de resíduos de couve (NS). A solarização aumentou a amplitude entre temperaturas máximas e mínimas nos tratamentos com e sem adição de matéria orgânica, quando comparado aos tratamentos não solarizados. Essas diferenças entre as temperaturas são condizentes
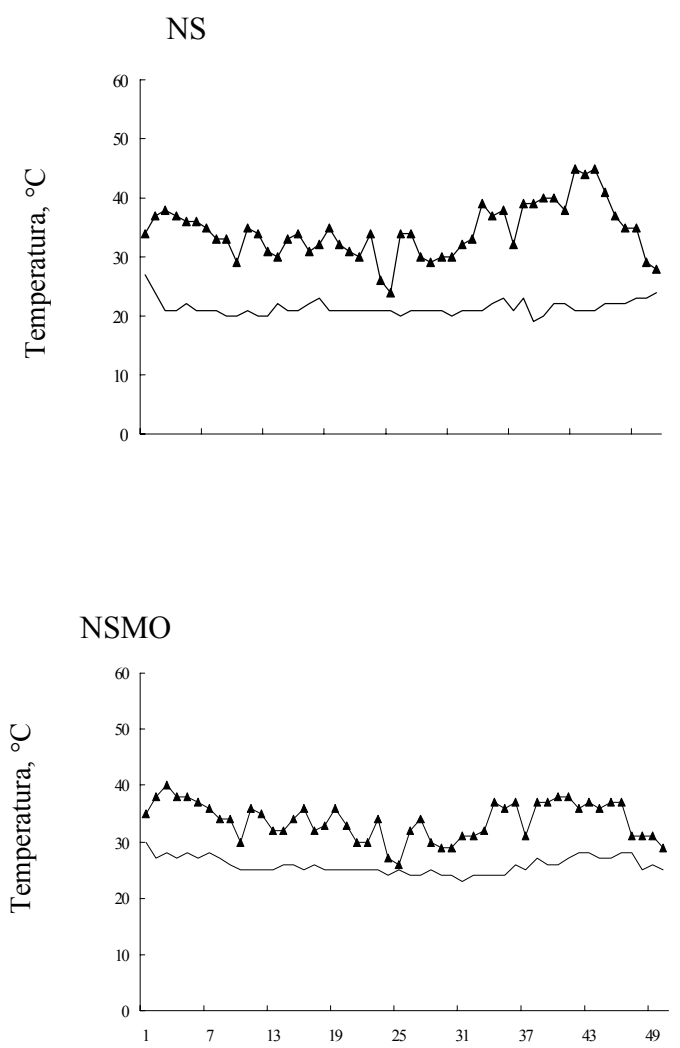

SMO

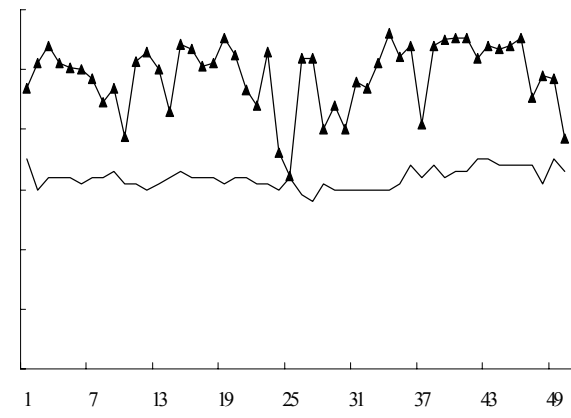

Dias de solarização

- Temperatura mínima - Temperatura máxima

Figura 2. Temperaturas máximas e mínimas no solo, em ${ }^{\circ} \mathrm{C}$, para os tratamentos sem solarização e sem incorporação de resíduos orgânicos (couve) (NS), com solarização e sem incorporação de resíduos orgânicos (couve) (S), sem solarização e com incorporação de resíduos orgânicos (couve) (NSMO) e com solarização e incorporação de resíduos orgânicos (couve) (SMO).

A matéria orgânica adicionada causou aumentos significativos dos índices de $\mathrm{pH}\left(\mathrm{CaCl}_{2}\right)$ somente para o tratamento SMO aos 7, 35 e 49 dias, sendo que aos 21 dias não houve diferença estatística entre este tratamento e o tratamento sem acréscimo de matéria orgânica e solarizado (S) (Tabela 1). A solarização foi responsável pelo aumento do $\mathrm{pH}$ no tratamento SMO durante todo o período de coleta, e para o tratamento sem adição de matéria orgânica, mas solarizado (S), somente aos 7 e 21 dias de solarização, quando comparado ao sem solarização e sem adição de matéria orgânica (NS).

Esses resultados diferem dos obtidos por Kaewruang, Sivasithamparam e Hardy (1989) que não obtiveram diferenças de $\mathrm{pH}$ entre solos apenas 
solarizados e não solarizados, sem adição de matéria orgânica, da Austrália, provavelmente pelo fato destes solos não terem sofrido acréscimo de matéria orgânica, bem como Grünzweig et al. (1999) que observaram redução de pH para solos solarizados em Israel. Entretanto, os resultados concordam com Patrício et al. (2006) que obtiveram pequenos aumentos nos valores de $\mathrm{pH}$ para áreas solarizadas em Mogi das Cruzes (SP).

Apenas o tratamento $\mathrm{S}$ apresentou significativas variações de $\mathrm{pH}$ entre as épocas de coleta de solo, sendo os valores de $\mathrm{pH}$ maiores no início, diminuindo gradativamente no transcorrer do experimento.

Tabela 1. Médias de valores de $\mathrm{pH}\left(\mathrm{CaCl}_{2}\right)$ de solo solarizado e não solarizado com ou sem adição de resíduos orgânicos (couve).

\begin{tabular}{|c|c|c|c|c|c|}
\hline \multirow[t]{3}{*}{ Solarização } & \multirow{3}{*}{$\begin{array}{l}\text { Resíduos orgânicos } \\
\text { (couve) }\end{array}$} & \multicolumn{4}{|c|}{ Épocas da coleta, dias } \\
\hline & & 7 & 21 & 35 & 49 \\
\hline & & \multicolumn{4}{|c|}{$\mathrm{pH}, \mathrm{CaCl}_{2}$} \\
\hline \multirow[t]{2}{*}{ Ausente } & Sem & $4,26 \mathrm{aB} \alpha^{(1)}$ & $4,16 \mathrm{aB} \alpha$ & $4,23 \mathrm{aA} \alpha$ & $4,10 \mathrm{aA} \alpha$ \\
\hline & Com & $4,23 \mathrm{aB} \alpha$ & $4,23 \mathrm{aB} \alpha$ & $4,36 \mathrm{aB} \alpha$ & $4,23 \mathrm{aB} \alpha$ \\
\hline \multirow[t]{2}{*}{ Presente } & Sem & $4,86 \mathrm{bA \alpha}$ & $4,66 \mathrm{bA} \alpha \beta \gamma$ & $4,33 \mathrm{bA} \gamma \beta$ & $4,13 \mathrm{bA} \gamma$ \\
\hline & Com & $5,20 \mathrm{aA} \alpha$ & $5,03 \mathrm{aA} \alpha$ & $5,33 \mathrm{aA} \alpha$ & $4,93 \mathrm{aA} \alpha$ \\
\hline
\end{tabular}

${ }^{1}$ Médias seguidas pela mesma letra não diferem entre si, pelo teste de Tukey, no nível de $5 \%$ de significância. Médias de três repetições. Para a comparação dos níveis de adição de resíduos orgânicos (couve) ao solo, fixados os níveis de solarização e épocas da coleta, utilizaram-se as minúsculas; para comparação dos níveis de solarização, fixados adição de resíduos orgânicos (couve) e épocas da coleta, utilizaram-se letras maiúsculas; para comparação das épocas de coletas de solo, fixados solarização e adição de resíduos orgânicos (couve), letras gregas.

O tratamento SMO apresentou maiores teores de matéria orgânica durante todo o experimento em decorrência da adição de resíduo vegetal de couve, quando comparado ao tratamento S (Tabela 2).

Em análises microbiológicas de solo, realizadas em complemento a este trabalho (CRUZ et al., 2005), os autores observaram maior atividade microbiana do solo no tratamento SMO, na segunda época de coleta (21 dias), o que pode explicar o fato da solarização ter aumentado significativamente os teores de matéria orgânica neste tratamento, provavelmente em decorrência da potencialização da ação desses microrganismos na degradação do material adicionado (couve).
De modo geral, pode-se observar que para o tratamento SMO houve aumento de matéria orgânica, em comparação à matéria orgânica adicionada inicialmente, e considerando os valores iniciais de matéria orgânica no solo $\left(14 \mathrm{~g} \mathrm{dm}^{-3}\right)$, o que sugere a interação de outros fatores bióticos e abióticos não quantificados neste trabalho, que podem ter sido favorecidos pela solarização.

Para os tratamentos solarizados ( $\mathrm{S}$ e SMO) houve redução de teor de matéria orgânica em função do tempo, porém somente para os tratamentos solarizados com adição de resíduos orgânicos (SMO) esta redução foi significativa, provavelmente em decorrência da estabilização da velocidade de mineralização da matéria orgânica recém adicionada (resíduos orgânicos de couve). 
Tabela 2. Médias de teores de matéria orgânica do solo $\left(\mathrm{g} \mathrm{dm}^{-3}\right)$ de solo solarizado e não solarizado com ou sem adição de resíduos orgânicos (couve).

\begin{tabular}{|c|c|c|c|c|c|}
\hline \multirow[t]{3}{*}{ Solarização } & \multirow{3}{*}{$\begin{array}{c}\text { Resíduos } \\
\text { orgânicos } \\
\text { (couve) }\end{array}$} & \multicolumn{4}{|c|}{ Épocas da coleta, dias } \\
\hline & & 7 & 21 & 35 & 49 \\
\hline & & \multicolumn{4}{|c|}{ M.O., $\mathrm{g} \mathrm{dm}^{-3}$} \\
\hline \multirow[t]{2}{*}{ Ausente } & Sem & $19,33 \mathrm{aA} \alpha^{(1)}$ & $17,00 \mathrm{aA} \alpha$ & $15,66 \mathrm{bA \alpha}$ & $15,66 \mathrm{aA} \alpha$ \\
\hline & Com & $20,66 \mathrm{aA} \alpha$ & $18,66 \mathrm{aB} \alpha$ & $19,00 \mathrm{aA} \alpha$ & $18,33 \mathrm{aA} \alpha$ \\
\hline \multirow[t]{2}{*}{ Presente } & Sem & $17,33 \mathrm{bA \alpha}$ & $16,66 \mathrm{bA \alpha}$ & $15,00 \mathrm{bA} \alpha$ & $13,00 \mathrm{bA} \alpha$ \\
\hline & Com & $22,66 \mathrm{aA} \alpha \beta$ & $25,00 \mathrm{aA} \alpha$ & $20,00 \mathrm{aA} \beta$ & $20,66 \mathrm{aA} \beta$ \\
\hline $\mathrm{CV}(\%)$ & 10,80 & & & & \\
\hline
\end{tabular}

${ }^{1}$ Médias seguidas pela mesma letra não diferem entre si, pelo teste de Tukey, no nível de $5 \%$ de significância. Médias de três repetições. Para a comparação dos níveis de adição de resíduos orgânicos (couve) ao solo, fixados os níveis de solarização e épocas da coleta, utilizaram-se as minúsculas; para comparação dos níveis de solarização, fixados adição de resíduos orgânicos (couve) e épocas da coleta, utilizaram-se letras maiúsculas; para comparação das épocas de coletas de solo, fixados solarização e adição de resíduos orgânicos (couve), letras gregas.

A matéria orgânica adicionada provocou aumento significativo na disponibilidade de fósforo somente na primeira época de coleta de solo (7 dias), para o tratamento SMO, não ocorrendo diferença significativa entre os tratamentos não solarizados. A técnica de solarização aumentou significativamente sua disponibilidade até os 21 dias para o tratamento SMO (Tabela 3) em aproximadamente nove vezes em relação ao valor inicial. O aumento da temperatura do solo pode ter favorecido o aumento do teor de fósforo para o tratamento $\mathrm{SMO}$, provocando uma elevação na atividade microbiana, como descrito anteriormente, principalmente no início da degradação desse material, diminuindo seus valores nas coletas posteriores, concordando com os resultados obtidos por Kwabiah et al. (2003), que por meio da análise de incorporação de diferentes materiais orgânicos ao solo também observaram diminuição nos valores de fósforo imediatamente depois do início das análises.

Provavelmente o aumento da atividade microbiana nesse tratamento fez com que o fósforo fosse temporariamente imobilizado pela biomassa microbiana para atender o crescimento da população de microrganismos (CRUZ et al., 2005; NOVAIS; SMYTH, 1999) ou então também pode ter sido precipitado em decorrência do aumento de $\mathrm{pH}$ nesses tratamentos (Tabela 1).

Segundo Mafongoya, Barak e Reed (2000) as maiores fontes de fósforo disponíveis para as plantas em solos tropicais são derivadas da mineralização da matéria orgânica recentemente adicionada ao solo. Yañes (2005) também observou aumento nos teores de fósforo, bem como potássio e nitrogênio para os tratamentos solarizados com acréscimo de matéria orgânica (cama de frango e torta de mamona).

O resíduo vegetal adicionado elevou significativamente os teores de potássio nos tratamentos SMO e NSMO (Tabela 4) havendo interação entre a adição do resíduo vegetal e a solarização. $\mathrm{O}$ efeito da solarização para potássio foi expressivo apenas para as amostras de solo do tratamento $\mathrm{SMO}$, até os 35 dias de experimento. $\mathrm{Na}$ coleta de solo aos 49 dias esses teores foram menores em relação aos verificados para o tratamento NSMO, provavelmente, em decorrência da degradação mais lenta do material orgânico, favorecendo maior disponibilidade de potássio somente aos 49 dias. 
Tabela 3. Médias dos teores de fósforo $\left(\mathrm{mg} \mathrm{dm}^{-3}\right)$ de solo solarizado e não solarizado com ou sem adição de resíduos orgânicos (couve).

\begin{tabular}{cccccc}
\hline Solarização & $\begin{array}{c}\text { Resíduos orgânicos } \\
\text { (couve) }\end{array}$ & $\mathbf{7}$ & $\mathbf{2 1}$ & $\mathbf{3 5}$ & $\mathbf{4 9}$ \\
& & \multicolumn{4}{c}{$\mathbf{P}, \mathbf{~ m g ~ d m}^{-3}$} \\
\hline Ausente & $\mathrm{Sem}$ & $3,33 \mathrm{aA} \alpha^{(1)}$ & $3,33 \mathrm{aA} \alpha$ & $3,00 \mathrm{aA} \alpha$ & $4,66 \mathrm{aA} \alpha$ \\
& $\mathrm{Com}$ & $5,33 \mathrm{aB} \alpha$ & $2,26 \mathrm{aB} \alpha$ & $1,36 \mathrm{aA} \alpha$ & $4,66 \mathrm{aA} \alpha$ \\
Presente & $\mathrm{Sem}$ & $11,33 \mathrm{bA} \alpha$ & $8,66 \mathrm{aA} \alpha$ & $13,33 \mathrm{aAA} \alpha$ & $3,66 \mathrm{aA} \alpha$ \\
& $\mathrm{Com}$ & $45,33 \mathrm{aA} \alpha$ & $18,66 \mathrm{aA} \beta$ & $4,83 \mathrm{aA} \beta$ & $6,66 \mathrm{aA} \beta$ \\
\hline $\mathrm{CV}(\%)$ & 103,18 & & &
\end{tabular}

${ }^{1}$ Médias seguidas pela mesma letra não diferem entre si, pelo teste de Tukey, no nível de $5 \%$ de significância. Médias de três repetições. Para a comparação dos níveis de adição de resíduos orgânicos (couve) ao solo, fixados os níveis de solarização e épocas da coleta, utilizaram-se as minúsculas; para comparação dos níveis de solarização, fixados adição de resíduos orgânicos (couve) e épocas da coleta, utilizaram-se letras maiúsculas; para comparação das épocas de coletas de solo, fixados solarização e adição de resíduos orgânicos (couve), letras gregas.

A elevação dos teores de potássio para o tratamento SMO a partir do primeiro momento de coleta pode ser explicada pelas temperaturas mais elevadas neste tratamento (Figura 2). Entretanto, a solarização não proporcionou aos tratamentos apenas solarizados (S) aumentos estatisticamente significativos nos teores de potássio, embora tenha sido observado relativo aumento deste elemento para estes tratamentos, quando comparados aos não solarizados sem resíduos orgânicos (NS) concordando com Patrício et al. (2006) e Grünzweig et al. (1999) que verificaram elevação nos teores de potássio e magnésio em solos solarizados, o que mostra a variação dos dados conforme as características iniciais de cada solo, sua localização e histórico de cultivo.

Tabela 4. Médias dos teores de potássio $\left(\mathrm{mmol}_{\mathrm{c}} \mathrm{dm}^{-3}\right)$ de solo solarizado e não solarizado com ou sem adição de resíduos orgânicos (couve).

\begin{tabular}{|c|c|c|c|c|c|}
\hline \multirow[t]{3}{*}{ Solarização } & \multirow{3}{*}{$\begin{array}{l}\text { Resíduos orgânicos } \\
\text { (couve) }\end{array}$} & \multicolumn{4}{|c|}{ Épocas da coleta, dias } \\
\hline & & 7 & 21 & 35 & 49 \\
\hline & & \multicolumn{4}{|c|}{$\mathbf{K}, \mathbf{m m o l}_{\mathbf{c}} \mathbf{d m}^{-3}$} \\
\hline \multirow[t]{2}{*}{ Ausente } & Sem & $0,86 \mathrm{bA} \alpha^{(1)}$ & $0,70 \mathrm{bA} \alpha$ & $0,53 \mathrm{aA} \alpha$ & $0,46 \mathrm{bA} \alpha$ \\
\hline & Com & $2,66 \mathrm{aB} \beta$ & $2,26 \mathrm{aB} \beta$ & $1,36 \mathrm{aB} \beta$ & 7,66 aA $\alpha$ \\
\hline \multirow[t]{2}{*}{ Presente } & Sem & $2,10 \mathrm{bA \alpha}$ & $1,10 \mathrm{bA} \alpha$ & $0,73 \mathrm{bA \alpha}$ & $0,60 \mathrm{bA \alpha}$ \\
\hline & Com & $6,53 \mathrm{aA} \alpha$ & $3,96 \mathrm{aA} \beta$ & $4,83 \mathrm{aA} \beta$ & $4,03 \mathrm{aB} \beta$ \\
\hline $\mathrm{CV}(\%)$ & 30,15 & & & & \\
\hline
\end{tabular}

${ }^{1}$ Médias seguidas pela mesma letra não diferem entre si, pelo teste de Tukey, no nível de 5\% de significância. Médias de três repetições. Para a comparação dos níveis de adição de resíduos orgânicos (couve) ao solo, fixados os níveis de solarização e épocas da coleta, utilizaram-se as minúsculas; para comparação dos níveis de solarização, fixados adição de resíduos orgânicos (couve) e épocas da coleta, utilizaram-se letras maiúsculas; para comparação das épocas de coletas de solo, fixados solarização e adição de resíduos orgânicos (couve), letras gregas. 
Durante todas as épocas de coleta de solo, os resíduos vegetais adicionados proporcionaram significativos aumentos na disponibilidade de nitrogênio total para o tratamento SMO e apenas nas duas primeiras épocas de coleta ( 7 e 21 dias) para o NSMO, tendendo a diminuir gradativamente até a última época de coleta de solo (Tabela 5) para ambos os tratamentos. A solarização foi responsável pelos maiores teores de nitrogênio total somente para os tratamentos $\mathrm{SMO}$ em todo o período.

Patrício et al. (2006) e Grünzweig et al. (1999) obtiveram resultados diferentes deste trabalho, no que se refere ao aumento nos valores de $\mathrm{N}$ em solos apenas solarizados, bem como Kaewruang, Sivasithamparam e Hardy (1989) que em solos apenas solarizados obtiveram aumentos nas concentrações de nitrogênio. Patrício (2001) também relata maiores teores deste elemento em solos solarizados, bem como maior produtividade de alface e controle de plantas daninhas.
A maior disponibilidade de nitrogênio no tratamento SMO, pode ser explicada pelas transformações microbianas, que favorecem a mineralização de nitrogênio através de enzimas produzidas pelos microrganismos, cujo crescimento está condicionado pela temperatura. Em contraste com a maioria das transformações microbianas as temperaturas ótimas para a mineralização do nitrogênio estão entre 40 e $60^{\circ} \mathrm{C}$ (ALEXANDER, 1967), ou seja, os mesmos intervalos de temperaturas obtidas neste trabalho para o tratamento SMO.

Ghini et al. (2003); Patrício (2001) e Sinigaglia et al. (2001) verificaram aumentos significativos de $\mathrm{NH}_{4}^{+}$decorrente da mineralização do nitrogênio orgânico do solo potencializado pelo aumento de temperatura, concordando com Stapleton (2000) que relata uma regularidade no aumento da concentração de nitrogênio em solos solarizados. Estes aumentos nos teores de nitrogênio em solos solarizados são, na maioria das vezes, os maiores responsáveis pelos aumentos de produção das culturas.

Tabela 5. Médias dos teores de Nitrogênio total $\left(\mathrm{g} \mathrm{kg}^{-1}\right)$ de solo solarizado e não solarizado com ou sem adição de resíduos orgânicos (couve).

\begin{tabular}{|c|c|c|c|c|c|}
\hline \multirow[t]{3}{*}{ Solarização } & \multirow{3}{*}{$\begin{array}{c}\text { Resíduos orgânicos } \\
\text { (couve) }\end{array}$} & \multicolumn{4}{|c|}{ Épocas da coleta, dias } \\
\hline & & 7 & 21 & 35 & 49 \\
\hline & & \multicolumn{4}{|c|}{$\mathbf{N}_{\text {total }}, \mathrm{g} \mathrm{kg}^{-1}$} \\
\hline \multirow[t]{2}{*}{ Ausente } & Sem & $0,53 \mathrm{bA} \alpha^{(1)}$ & $0,53 \mathrm{bA} \alpha$ & $0,54 \mathrm{aA} \alpha$ & $0,53 \mathrm{aA} \alpha$ \\
\hline & Com & $0,63 \mathrm{aB} \alpha$ & $0,66 \mathrm{aB} \alpha$ & $0,59 \mathrm{aB} \alpha \beta$ & $0,52 \mathrm{aB} \beta$ \\
\hline \multirow[t]{2}{*}{ Presente } & Sem & $0,57 \mathrm{bA} \alpha$ & $0,50 \mathrm{bA} \alpha$ & $0,48 \mathrm{bA} \alpha$ & $0,52 \mathrm{bA} \alpha$ \\
\hline & Com & $0,94 \mathrm{aA} \alpha$ & $0,81 \mathrm{aA} \beta$ & $0,72 \mathrm{aA} \gamma \beta$ & $0,69 \mathrm{aA} \gamma$ \\
\hline
\end{tabular}

${ }^{1}$ Médias seguidas pela mesma letra não diferem entre si, pelo teste de Tukey, no nível de 5\% de significância. Médias de três repetições. Para a comparação dos níveis de adição de resíduos orgânicos (couve) ao solo, fixados os níveis de solarização e épocas da coleta, utilizaram-se as minúsculas; para comparação dos níveis de solarização, fixados adição de resíduos orgânicos (couve) e épocas da coleta, utilizaram-se letras maiúsculas; para comparação das épocas de coletas de solo, fixados solarização e adição de resíduos orgânicos (couve), letras gregas.

O resíduo vegetal aplicado também proporcionou diferenças significativas nos teores de cálcio (Tabela 6). No tratamento SMO a matéria orgânica adicionada aumentou a disponibilidade deste nutriente na primeira época de coleta. Entretanto a disponibilidade de cálcio foi prejudicada pela adição de matéria orgânica nos primeiros momentos de coleta (7 e 21 dias), verificada no tratamento NSMO, em comparação ao não solarizado sem adição de resíduos orgânicos de couve (NS), provavelmente devido à complexação do cálcio pela mesma. 
A solarização proporcionou aumentos nos teores cálcio, somente até a segunda época de coleta, para o tratamento SMO, não diferindo dos demais tratamentos após este período. Os tratamentos NS, S e SMO apresentaram significativa diminuição na disponibilidade de cálcio durante as avaliações.

Há alguns anos que autores observam a relação de cálcio com outros elementos químicos de solo, como Mascarenhas (1977) que relatou o fato de solos com elevados teores de matéria orgânica, em geral, apresentarem maiores teores de cálcio total, e estes poderem ser adsorvidos pelo complexo coloidal do solo. Estes relatos podem explicar os resultados obtidos neste trabalho, pois à medida que a saturação de bases aumenta, os teores de cálcio também aumentam em decorrência do aumento de $\mathrm{pH}$, como foi observado para o tratamento SMO, reafirmando a estreita relação existente entre $\mathrm{pH}$ (Tabela 1) e cálcio.

Para o tratamento SMO, as altas temperaturas e a adição de matéria orgânica fresca podem ter favorecido a atividade da microbiota do solo, como observado no trabalho de Cruz et al., (2005) e dessa forma, a matéria orgânica adicionada (resíduo de couve) provavelmente foi mais rapidamente decomposta por meio da ação dos microrganismos, favorecendo a disponibilidade de cálcio, principalmente na primeira coleta de solo. Os resultados concordam com Stapleton (2000) que relata a não regularidade de aumento nos teores de cálcio em solos solarizados.

Tabela 6. Médias dos teores de cálcio $\left(\mathrm{mmol}_{\mathrm{c}} \mathrm{dm}^{-3}\right)$, de solo solarizado e não solarizado com ou sem adição de resíduos orgânicos (couve).

\begin{tabular}{|c|c|c|c|c|c|}
\hline \multirow[t]{3}{*}{ Solarização } & \multirow{3}{*}{$\begin{array}{l}\text { Resíduos orgânicos } \\
\text { (couve) }\end{array}$} & \multicolumn{4}{|c|}{ Épocas da coleta, dias } \\
\hline & & 7 & 21 & 35 & 49 \\
\hline & & \multicolumn{4}{|c|}{$\mathrm{Ca}, \mathbf{m m o l}_{\mathbf{c}} \mathbf{d m}^{-3}$} \\
\hline \multirow[t]{2}{*}{ Ausente } & Sem & $25,33 \mathrm{aA} \alpha^{(1)}$ & $9,66 \mathrm{aA} \alpha \beta$ & $8,33 \mathrm{aA} \alpha$ & $9,33 \mathrm{aA} \beta$ \\
\hline & Com & $10,66 \mathrm{bB} \alpha$ & $11,66 \mathrm{bB} \alpha$ & $10,33 \mathrm{aA} \alpha$ & $7,66 \mathrm{aA} \alpha$ \\
\hline \multirow[t]{2}{*}{ Presente } & Sem & $25,33 \mathrm{bA \alpha}$ & $17,66 \mathrm{aA} \alpha \beta$ & $12,00 \mathrm{aA} \alpha \beta$ & $7,66 \mathrm{aA} \beta$ \\
\hline & Com & $44,66 \mathrm{aA} \alpha$ & $32,00 \mathrm{aA} \alpha \beta$ & $22,00 \mathrm{aA} \beta$ & $16,66 \mathrm{aA} \beta$ \\
\hline $\mathrm{CV}(\%)$ & 43,19 & & & & \\
\hline
\end{tabular}

${ }^{1}$ Médias seguidas pela mesma letra não diferem entre si, pelo teste de Tukey, no nível de $5 \%$ de significância. Médias de três repetições. Para a comparação dos níveis de adição de resíduos orgânicos (couve) ao solo, fixados os níveis de solarização e épocas da coleta, utilizaram-se as minúsculas; para comparação dos níveis de solarização, fixados adição de resíduos orgânicos (couve) e épocas da coleta, utilizaram-se letras maiúsculas; para comparação das épocas de coletas de solo, fixados solarização e adição de resíduos orgânicos (couve), letras gregas.

A adição dos resíduos orgânicos de couve ao solo aumentou significativamente os teores de magnésio no tratamento NSMO até a segunda época de coleta de solo, e para o tratamento SMO os aumentos foram significativamente maiores em todas as épocas de coleta (Tabela 7).

A solarização elevou os teores de magnésio somente no tratamento SMO. Para este tratamento, os teores de magnésio decresceram significativamente até o final das coletas. Para o tratamento $S$, que não recebeu matéria orgânica, a solarização não promoveu aumento significativo dos teores deste nutriente quando comparado ao NS, ao contrário dos resultados obtidos por Grünzweig et al. (1999), que verificaram elevação nos teores de magnésio em solos apenas solarizados, mostrando a variação dos resultados conforme as condições climáticas, as regiões geográficas e as características iniciais dos solos envolvidos. 
Tabela 7. Médias dos teores de magnésio $\left(\mathrm{mmol}_{\mathrm{c}} \mathrm{dm}^{-3}\right)$ de solo solarizado e não solarizado com ou sem adição de resíduos orgânicos (couve).

\begin{tabular}{|c|c|c|c|c|c|}
\hline \multirow[t]{3}{*}{ Solarização } & \multirow{3}{*}{$\begin{array}{c}\text { Resíduos orgânicos } \\
\text { (couve) }\end{array}$} & \multicolumn{4}{|c|}{ Épocas da coleta, dias } \\
\hline & & 7 & 21 & 35 & 49 \\
\hline & & \multicolumn{4}{|c|}{$\mathrm{Mg}, \mathbf{m m o l}_{\mathrm{c}} \mathbf{d m}^{-3}$} \\
\hline \multirow[t]{2}{*}{ Ausente } & Sem & $3,33 \mathrm{bA} \alpha^{(1)}$ & $2,33 \mathrm{bA} \alpha$ & $2,00 \mathrm{aA} \alpha$ & $2,33 \mathrm{aA} \alpha$ \\
\hline & Com & $5,00 \mathrm{aB} \alpha$ & $6,00 \mathrm{aB} \alpha$ & $4,66 \mathrm{aB} \alpha$ & $3,33 \mathrm{aB} \alpha$ \\
\hline \multirow[t]{2}{*}{ Presente } & Sem & $6,33 \mathrm{bA \alpha}$ & $4,66 \mathrm{bA} \alpha$ & $3,66 \mathrm{bA \alpha}$ & $3,00 \mathrm{bA} \alpha$ \\
\hline & Com & 18,33 aA $\alpha$ & 13,33 aA $\beta$ & 9,66 aA $\beta \gamma$ & $9,00 \mathrm{aA} \gamma$ \\
\hline CV(\%) & 32,12 & & & & \\
\hline
\end{tabular}

${ }^{1}$ Médias seguidas pela mesma letra não diferem entre si, pelo teste de Tukey, no nível de $5 \%$ de significância. Médias de três repetições. Para a comparação dos níveis de adição de resíduos orgânicos (couve) ao solo, fixados os níveis de solarização e épocas da coleta, utilizaram-se as minúsculas; para comparação dos níveis de solarização, fixados adição de resíduos orgânicos (couve) e épocas da coleta, utilizaram-se letras maiúsculas; para comparação das épocas de coletas de solo, fixados solarização e adição de resíduos orgânicos (couve), letras gregas.

Os resíduos orgânicos adicionados (couve) reduziram significativamente o alumínio trocável para o tratamento NSMO aos 49 dias do experimento e a partir de 21 dias para o tratamento SMO (Tabela 8). A solarização foi responsável pela redução significativa nas duas primeiras épocas de coleta do solo (7 e 21 dias) para o tratamento S e para o SMO durante todos o período.
$\mathrm{O}$ aumento do índice de $\mathrm{pH}$ do solo pode ter provocado a redução dos teores de alumínio para o tratamento SMO. Coutinho, Natale e Souza (1993) relatam que solos com $\mathrm{pH}$ mais elevados apresentam menores disponibilidades de alumínio em decorrência de sua capacidade de precipitação nessas circunstâncias. Segundo Raij (1993) a matéria orgânica presente no solo é capaz de complexar o alumínio, reduzindo seus efeitos deletérios nas culturas.

Tabela 8. Médias dos teores de alumínio $\left(\mathrm{mmol}_{\mathrm{c}} \mathrm{dm}^{-3}\right)$, de solo solarizado e não solarizado com ou sem adição de resíduos orgânicos (couve).

\begin{tabular}{|c|c|c|c|c|c|}
\hline \multirow[t]{3}{*}{ Solarização } & \multirow{3}{*}{$\begin{array}{c}\text { Resíduos orgânicos } \\
\text { (couve) }\end{array}$} & \multicolumn{4}{|c|}{ Épocas da coleta, dias } \\
\hline & & 7 & 21 & 35 & 49 \\
\hline & & \multicolumn{4}{|c|}{$\mathrm{Al}, \mathbf{m m o l}_{\mathbf{c}} \mathbf{d m}^{-3}$} \\
\hline \multirow[t]{2}{*}{ Ausente } & Sem & $3,33 \mathrm{aA} \beta^{(1)}$ & $4,00 \mathrm{aA} \alpha \beta$ & $3,33 \mathrm{aA} \beta$ & $5,00 \mathrm{aA} \alpha$ \\
\hline & Com & $3,33 \mathrm{aA} \alpha$ & $3,00 \mathrm{aA} \alpha$ & $2,66 \mathrm{aA} \alpha$ & $3,33 \mathrm{bA \alpha}$ \\
\hline \multirow[t]{2}{*}{ Presente } & Sem & $0,33 \mathrm{aB} \gamma$ & $1,33 \mathrm{aB} \alpha \gamma$ & $2,66 \mathrm{aA} \alpha \beta$ & $4,00 \mathrm{aA} \alpha$ \\
\hline & Com & $0,33 \mathrm{aB} \alpha$ & $0,02 \mathrm{bB} \alpha$ & $0,02 \mathrm{bB} \alpha$ & $0,33 \mathrm{bB} \alpha$ \\
\hline
\end{tabular}

$\mathrm{CV}(\%) \quad 31,20$

${ }^{1}$ Médias seguidas pela mesma letra não diferem entre si, pelo teste de Tukey, no nível de $5 \%$ de significância. Médias de três repetições. Para a comparação dos níveis de adição de resíduos orgânicos (couve) ao solo, fixados os níveis de solarização e épocas da coleta, utilizaram-se as minúsculas; para comparação dos níveis de solarização, fixados adição de resíduos orgânicos (couve) e épocas da coleta, utilizaram-se letras maiúsculas; para comparação das épocas de coletas de solo, fixados solarização e adição de resíduos orgânicos (couve), letras gregas. 
A solarização com acréscimo de resíduo vegetal de fácil decomposição como resíduos de orgânicos de couve (baixa relação $\mathrm{C} / \mathrm{N}(15,8))$, proporcionam ao solo características positivas para o manejo da maioria das culturas (ciclos curtos e longos), onde há grande exigência quanto à disponibilidade de nutrientes no início de seu desenvolvimento e a solarização, além de controlar fitopatógenos, plantas daninhas e alguns insetos, também pode contribuir com a disponibilização de minerais presentes em resíduos orgânicos de culturas incorporadas ao solo.

\section{Conclusões}

A solarização com incorporação de resíduos orgânicos (couve), tratamento SMO, aumentou os índices de $\mathrm{pH}$ e os teores de matéria orgânica, fósforo, nitrogênio total, potássio, cálcio e magnésio, e reduziu os teores de alumínio.

\section{Referências}

ALEXANDER, M. Introduction to soil microbiology. 4.ed. New York: John Wiley, 1967.

AMBRÓSIO, M. M. Q.; BUENO, C. J.; SOUZA, N. L. Sobrevivência de Macrophomina phaseolina em solo incorporado com brócolos seguido de solarização. Summa Phytopathologica, Botucatu, v. 30, n. 3, p. 364-370, 2004.

BUENO, C. J.; AMBROSIO, M. M. Q.; SOUZA, N. L.; CEREZINE, P. C. Controle de Fusarium oxysporum f. sp. lycopersici raça 2, Macrophomina phaseolina e Sclerotium rolfsii em microcosmo simulando solarização com prévia incorporação de couve. Summa Phytopathologica, Botucatu, v. 30, n. 3, p. 356-363, 2004.

BUENO, C. J.; SOUZA, N. L. Impacto da solarização de solo sobre o antagonismo de Pseudomonas spp. fluorescente à Rhizoctonia solani Küln G A 4HGI. Summa Phytopathologica, Botucatu, v. 29, n. 2, p. 147-152, 2003.

CARVALHO, W. A.; ESPINDOLA, C. R.; PACCOLA, A. A. Levantamento de solos da Fazenda Lageado - Estação Experimental Presidente Médice. Botucatu: Faculdade de Ciências Agronômicas da UNESP, 1983. (Boletim Científico, n. 1).

COUTINHO, E. L. M; NATALE, W.; SOUZA, E. C. A. Adubos e corretivos: aspectos particulares na olericultura. In: FERREIRA, M. E.; CASTELLANE, P. D.; CRUZ, M. C.
P.(Ed.). Nutrição e adubação de hortaliças. Piracicaba: Associação Brasileira para Pesquisa da Potassa e do Fosfato, 1993. p.85-140.

CRUZ, J. C. S. Aspectos microbiológicos e químicos em solo submetido à solarização. 2003. Dissertação (Mestrado em Proteção de Plantas) - Faculdade de Ciências Agronômicas,Universidade Estadual Paulista, Botucatu.

CRUZ, J. C. S.; ROCHA, M. M.; SOUZA, N. L.; PADOVANI, C. R.; MINHONI, M. T. A. Aspectos microbiológicos de solo e a técnica de solarização. Summa Phytopathologica, Botucatu, v. 31, n. 1, p. 74-81, 2005.

EMPRESA BRASILEIRA DE PESQUISA AGROPECUÁRIA - EMBRAPA. Centro Nacional de Pesquisa de Solos. Sistema brasileiro de classificação de solos. Rio de Janeiro: EMBRAPA, 1999.

GAMLIEL, A.; AUSTERWEIL, M.; KRITZMAN, G. Nonchemical approach to soilborne pest management - organic amendments. Crop Protection, Cotonou, v. 19, n. 8-10, p. $847-853,2000$.

GHINI, R.; SCHOENMAKER, I. A. S.; BETTIOL, W. Solarização do solo e incorporação de fontes de matéria orgânica no controle de Pythium spp. Pesquisa Agropecuária Brasileira, Brasília, v. 37, n. 9, p. 1253-1261, 2002.

GHINI, R.; PATRICIO ,F. R. A.; SOUZA, M. D.; SINIGAGLIA, C; BARROS, B. C.; LOPES, M. E. B. M.; TESSARIOLI NETO, J.; CANTARELLA, H. Efeito da solarização sobre propriedades físicas, químicas e biológicas de solos. Revista Brasileira de Ciência do Solo, Viçosa, v. 27, n. 1, p. 71-79, 2003.

GRÜNZWEIG, J. M.; KATAN, J.; BEM-TAL, Y.; RABINOWITCH, H. D. The role of mineral nutrients in the increased growth response of tomato plants in solarized soil. Plant and Soil, Nehterlands, v. 206, n. 1, p. 21-27, 1999.

KAEWRUANG, W.; SIVASITHAMPARAM, K.; HARDY, G. E. Use of soil solarization to control roots rots in gerberas (Gerbera jamesonii). Biology and Fertility of Soils, Firenze, v. 8, n. 1, p. 38-47, 1989.

KATAN, J. Solar heating (solarization) of soil for control of soil borne pests. Annual Review of Phytopathology, Palo Alto, v. 19, p. 211-236, 1981.

KATAN, J.; DEVAY, E. J. Soil solarization. London: CRC Press, 1991.

KUVA, M. A.; ALVES, P. L. C. A.; ERASMO, E. L. A. Efeitos da solarização do solo através de plástico transparente sobre o desenvolvimento da tiririca (Cyperus rotundus). Planta Daninha, Jaboticabal, v. 13, n. 1, 1995. 
KWABIAH, A. B.; PALM, C. A.; STOSKOPF, N. C.; VORONEY, R. P. Response of soil microbial biomass dynamics to quality of plant materials with emphasis on $\mathrm{P}$ availability. Soil Biology and Biochemistry, Oxford, v. 35, n. 2, p. 207-216, 2003.

MAlAVOltA, E.; VITTI, G. G.; OliveirA, S. A. Metodologia para análise de elementos em material vegetal. In: _. Avaliação do estado nutricional das plantas: princípios e aplicações. 2. ed. Piracicaba: Associação Brasileira para Pesquisa da Potassa e do Fosfato (Potafós), 1997. p. 231-315.

MARQUE, J. M.; SOUZA, N. L.; CUTOLO-FILHO, A. A. Efeito da solarização do solo na sobrevivência de Phytophthora capsici em cultivo protegido. Fitopatologia Brasileira, Brasília, v. 27, n. 1, p.42-47, 2002.

MASCARENHAS, H. A. A. Cálcio, enxofre ferro no solo e na planta. Campinas: Fundação Cargill, 1977, 95p.

MAFONGOYA, P. L.; BARAK, P.; REED, J. D. Carbon, nitrogen and phosphorus mineralization of tree leaves and manure. Biology and Fertility of Soils, Firenze, v. 30, n. 4, p. 298-305, 2000.

MONTEGOMERY, D. C. Design and analysis of experiments. 3.ed. New York: John Wiley, 1991.

NOVAIS, R. F.; SMYTH, T. J. Fósforo em solo e planta em condições tropicais. Viçosa: UFV/DPS, 1999.

OTIENO, W.; TERMORSHUIZEN, A; JEGER, M.; OTHIENO, C. O. Efficacy of soil solarization, Trichoderma harzianum, and coffee pulp amendment against Armilaria spp. Crop Protection, Cotonou, v. 22, n. 2, p. 325-331, 2003.

PATRÍCIO, F. R. A. Solarização do solo no controle de fitopatógenos. In: REUNIÃO ITINERANTE DE FITOSSANIDADE DO INSTITUTOBIOLÓGICO. 4., 2001, Ribeirão Preto, Anais... Ribeirão Preto: Instituto Biológico, 2001.p.167-171.

Solarização de solo para as culturas de alface, cenoura e morango. Summa Phytopathologica, Botucatu, v. 30, n. 1, p. 140-142, 2004.

PATRÍCIO, F. R. A; SINIGAGLIA, C.; BARROS, B. C.; FREITAS, S. S.; TESSARIOLI NETO, J.; CANTARELLA, H.; GHINI, R. Solarization and fungicides for the control of crop, bottom, rot and weeds in lettuce. Crop Protection, Cotonou, v. 25, n. 1, p. 31-38, 2006.
RAIJ, B. V. Princípios de correção e de adubação para mudas e para produção comercial. In: FERREIRA, M. E.; CASTELLANE, P. D.; CRUZ, M. C. P. (Ed.). Nutrição e Adubação de Hortaliças. Piracicaba: Associação Brasileira para Pesquisa da Potassa e do Fosfato, 1993. p. $75-84$.

RAIJ, B. V.; ANDRADE, J. C.; CANTARELLA, H.; QUAGGIO, J. A. (Ed.) Análise química para avaliação da fertilidade de solos tropicais. Campinas: Instituto Agronômico, 2001.

RICCI, M. S. F.; ALMEIDA, D. L.; FERNANDES, M. C. A.; RIBEIRO, R. L. D.; CANTANHEIDE, M. C. S. Efeitos da solarização do solo na densidade populacional da tiririca e na produtividade de hortaliças sob manejo orgânico. Pesquisa Agropecuária Brasileira, Brasília, v. 35, n. 11, p. 2175-2179, 2000.

ROSA, E. A.; HEANEY, R. K.; FENWICK, G. R. Glucosinolates in crop plants. Horticultural Reviews, New York, v. 19, p. 99-215, 1997.

STAPLETON, J. J.; DEVAY, J. E. Soil solarization: a non chemical approach for management of plant pathogens and pests. Crop Protection, Cotonou, v. 5, n. 3, p. 190198, 1986.

Soil solarization; a natural mechanism of integrated pest management. In: _______. Novel approaches to integrated pest management. Boca Raton: Lewis Publishing, 1995. p. 309-322

STAPLETON, J. J. Soil solarization in various agricultural production systems. Crop Protection, Cotonou, v. 19, n. 8-10, p. 837-841, 2000.

SINIGAGLIA, C.; PATRÍCIO, F. R. A.; GHINI, R.; MALAVOLTA, V. M. A.; TESSARIOLI, J.; FREITAS, S. S. Controle de Sclerotinia minor, Rhizoctonia solani e plantas daninhas de alface pela solarização do solo e sua integração com controle químico. Summa Phytopathologica, Botucatu, v. 27, n. 2, p. 229-235, 2001.

SOUZA, N. L.; BUENO, C. J. Sobrevivência de clamidósporos de Fusarium oxysporum f.sp. lycopersici raça 2 e escleródios de Sclerotium rolfsii em solo solarizado incorporado com matéria orgânica. Summa Phytopathologica, Botucatu, v. 29, n. 2, p. 153-160, 2003.

YAÑES, L. D. T. Resistência genética, fungicidas e solarização para o controle de Thielaviopsis basicola (Berk \& Broome) Ferraris na cultura de alface (Lactuca sativa L.). 2005. Tese (Doutorado em Agronomia) - Escola Superior de Agricultura Luiz de Queiroz, Piracicaba. 
